Nicole Kusserow und Pit Oertel

\title{
Ein Schmökerabend als neues Format für die Lange Nacht einer wissenschaftlichen Bibliothek
}

Zusammenfassung: Mit dem Schmökerabend soll ein neues Veranstaltungsformat speziell für wissenschaftliche Bibliotheken, wie der Hochschulbibliothek der TH Wildau, geschaffen werden. Diese Art des Zusammenkommens von Jung und Alt und des gemeinschaftlichen Lesens findet in der Regel in öffentlichen Bibliotheken statt. Im Fokus soll die Hochschulbibliothek mit diesem Event als frei zugängliche und öffentliche Bildungseinrichtung stehen. Mit einem gutem Konzept unter einem vorgeschlagenen Motto kann diese Veranstaltung für jede Bibliothek ein Mehrwert sein.

Schlüsselwörter: Veranstaltung, Hochschulbibliothek, Bildung, Kultur

\section{Reading Nights as New Format for Long Night Events at Research Libraries}

Abstract: A new event format aiming at research and academic libraries such as the library of the Technical University of Applied Sciences in Wildau is supposed to be created with the introduction of the reading night. This kind of event bringing together people of all ages for joint reading is usually found in public libraries. As to research libraries the idea is to present them as generally accessible and public educational institutions. With a well elaborated concept following a motto agreed upon beforehand a reading night can mean an additional benefit for any of the libraries involved.

Keywords: Event; Academic library; Education; Culture

Nicole Kusserow: nicole.kusserow@th-wildau.de

Pit Oertel: pit.oertel@th-wildau.de

Zum dritten Mal in Folge bot die Hochschulbibliothek der Technischen Hochschule Wildau (FH) ihre 1.400 qm für einen Schmökerabend an, bei dem um die 80 Gäste begrüßt werden konnten. Vom Samstag, dem 30. November 2013, 17 Uhr, bis zum Sonntag, dem 1. Dezember, $11 \mathrm{Uhr}$, verwandelte das Team der Hochschulbibliothek die „Bibliothek des Jahres 2012“ in einen großen Lesesaal. Diese Form 
der Langen Nacht bietet sich an, da sich der Bibliotheksraum um einen überdachten Innenhof über drei Etagen verteilt, gut überschaubar ist und zudem auch Nischen bereithält, um die benötigten ca. 50 Feldbetten aufzustellen.

Aufbauend auf den Erfahrungen der beiden vorangegangenen Schmökerabende, verfolgte die Veranstaltung das Ziel, dieses Format fortzusetzen und als festen Programmpunkt der Hochschulbibliothek zu verankern. Den Besuchern soll die Wahrnehmung der Bibliothek als öffentliche Bildungseinrichtung nahe gebracht werden.

Der erste Abend galt als Testlauf und aufgrund dessen guter Resonanz fand ein zweiter mit gewachsenem interessiertem Publikum statt. Zum dritten Schmökerabend bestand das Projektteam aus den Auszubildenden der Hochschulbibliothek, einem Projektmitarbeiter und den studentischen Hilfskräften. Mehr als 100 Arbeitsstunden wurden für die Vorbereitungen aufgewendet.

Um den dritten Schmökerabend umfangreicher zu gestalten, wurden die Programmpunkte erweitert. Da die Nachtwanderung beim zweiten Schmökerabend sehr gut bei den Besuchern angekommen war, wurde dieses Jahr nicht darauf verzichtet. Eine Kollegin der Stadtbibliothek Wildau, Frau Kerstin Nielsen, leitete das Bilderbuchkino „Die fantastischen fliegenden Bücher des Morris Lessmore“ im Haus der Hochschulbibliothek ein. Zur Einführung in die Geschichte, die von fliegenden Büchern handelt, gab es als visuelle Untermalung ausgelegte Buchstaben. Die Buchstaben luden den ganzen Abend zu neuen Wortspielen ein, so wurde beispielsweise im Laufe des Abends ein Liebesbekenntnis für eine Silke gelegt und allen Übernachtenden wurde auf diese Weise ein „Guten Morgen“ gewünscht.

Nach der oben beschriebenen Einführung wanderte knapp die Hälfte der Besucher verschiedenen Alters in der Dämmerung zur Stadtbibliothek Wildau und verfolgte dort das Bilderbuchkino. Zum Abschluss der Geschichte wurde der dazugehörige Kurzfilm gezeigt. Es bestand die Möglichkeit, ein Medium aus der Stadtbibliothek auszuleihen und zum Schmökern in die Hochschulbibliothek mitzunehmen.

Neu hinzu kam die Idee einer Schreibwerkstatt, in der sich die Besucher an neuen Formen des Schreibens versuchen konnten, z.B. verschiedene Gedichtformen wie Haiku oder auch Elfchen. Selbstverständlich konnten auch eigene Geschichten erfunden und verfasst werden. Die Kinder brachten ihre Fantasien und Ideen zu Papier. Der Kreativität waren keine Grenzen gesetzt. So entstand u.a. von einer jungen Besucherin ein Fantasierezept eines Kuchens, der für mehr als 500 Leute gereicht hätte.

Großen Anklang fand auch die diesjährige Bastelecke, wo ein reges Interesse an Fröbelsternen entstand. Den ganzen Abend über wurden ca. 150 Sterne gefaltet, wobei der erste meistens misslang, aber die Hilfe der Mitarbeiter und 
anderer Bastler zu einem wunderschönen zweiten, dritten und auch weiteren Stern führte. Dabei unterstützten sich die jüngeren und älteren Besucher gegenseitig beim Falten.

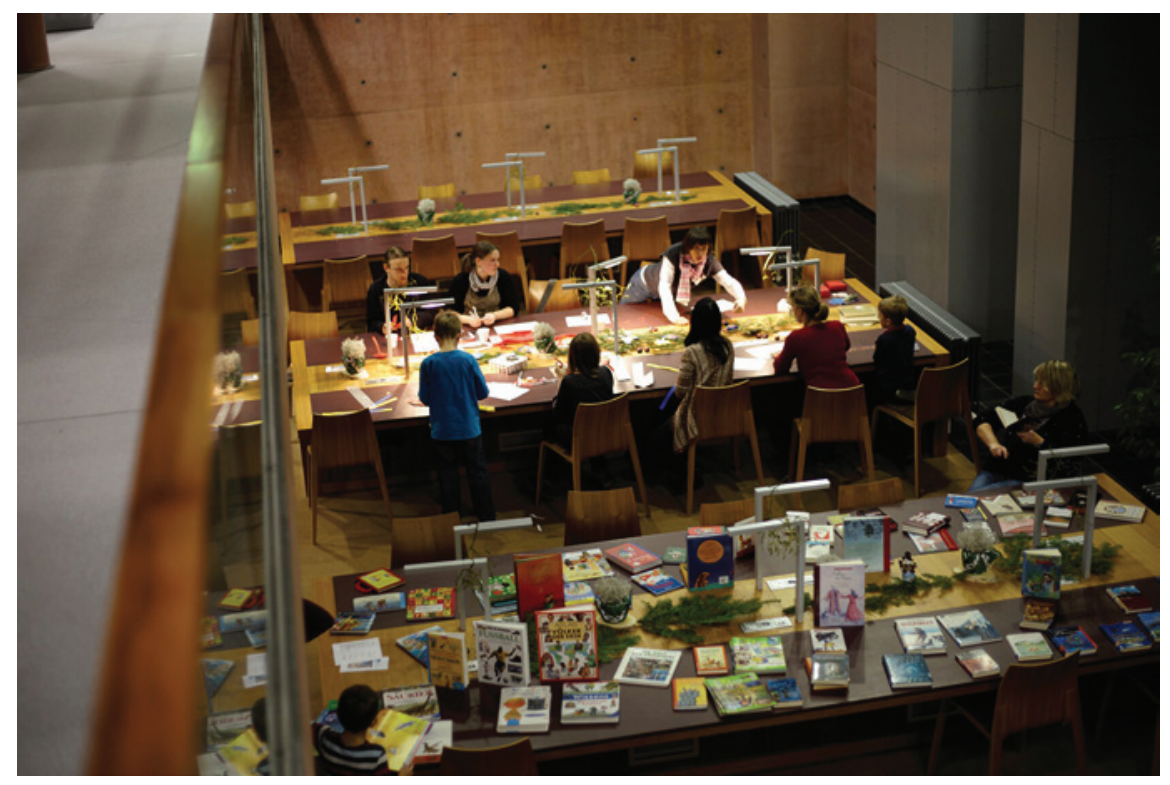

Abb. 1: Die Bücherauswahl und die Bastelecke.

Ein weiteres Programmhighlight waren zwei Lesungen, die im Schnitt von jeweils zehn Leuten wahrgenommen wurden. Sie lauschten gespannt den Werken „Emil und die Detektive“ von Erich Kästner und dem selbst verfassten Werk „Tag der Nacht" von Marcel Fenske-Pogrzeba.

Wie in den Vorjahren fand ein Quiz für Jung und Alt in der Räumlichkeit der Mediathek statt. Die besten Fünf mit den meisten richtigen Antworten bekamen verschiedene Preise, wie z.B. ausgewählte Bücher, Tassen und USB-Sticks der TH Wildau.

Um das Motto zu festigen, beschlossen wir gemeinsam zum dritten Schmökerabend, dass die Bibliothek zur internetfreien Zone erklärt wird. So konnte sich der Besucher ganz der ausgewählten Literatur und dem Miteinander widmen.

Die Realisierung des geplanten Konzepts sollte durch Sponsoring der Veranstaltung organisiert werden, da finanzielle Mittel nicht bereitstanden und kein Eintritt für die Gäste erhoben werden sollte. Die Förderer für das Catering und die Feldbetten waren neben dem hochschuleigenen Förderverein die Klinikum Dahme-Spreewald GmbH und das DRK Fläming-Spreewald. Kooperationspartner 
im bibliothekarischen Umfeld waren zudem die Gemeindebibliotheken aus Eichwalde und Zeuthen sowie die Stadtbibliotheken Wildau und Königs Wusterhausen, die Bücherspenden, Gesellschaftsspiele, Quizmaterialien und Dekorationsmaterialien zur Verfügung stellten.

Um gegebenenfalls finanzielle Mittel für die geplanten Programmpunkte zu erhalten, wurde ein jeweils individuelles Sponsoringanschreiben verfasst. Interesse bestand für diese Veranstaltung seitens einiger Verlage und Buchhandlungen, die eine Auswahl ihrer Produkte als Sachmittelspenden schickten. Diese wurden für ausgewählte Programmpunkte wie z.B. für die Quizveranstaltung verwendet und für die Besucher zum Schmökern ausgelegt.

Die drei Ebenen der Bibliothek wurden effektiv genutzt, um die Auswahl der Materialien und der verschiedenen Medien für jede Generation optimal zu präsentieren. So fanden sich für die interessierten Gäste Kinder- und Jugendliteratur, Erwachsenen- sowie Weihnachtsliteratur übersichtlich dargestellt und auch der Bestand der Hochschulbibliothek war für jedermann zugänglich.

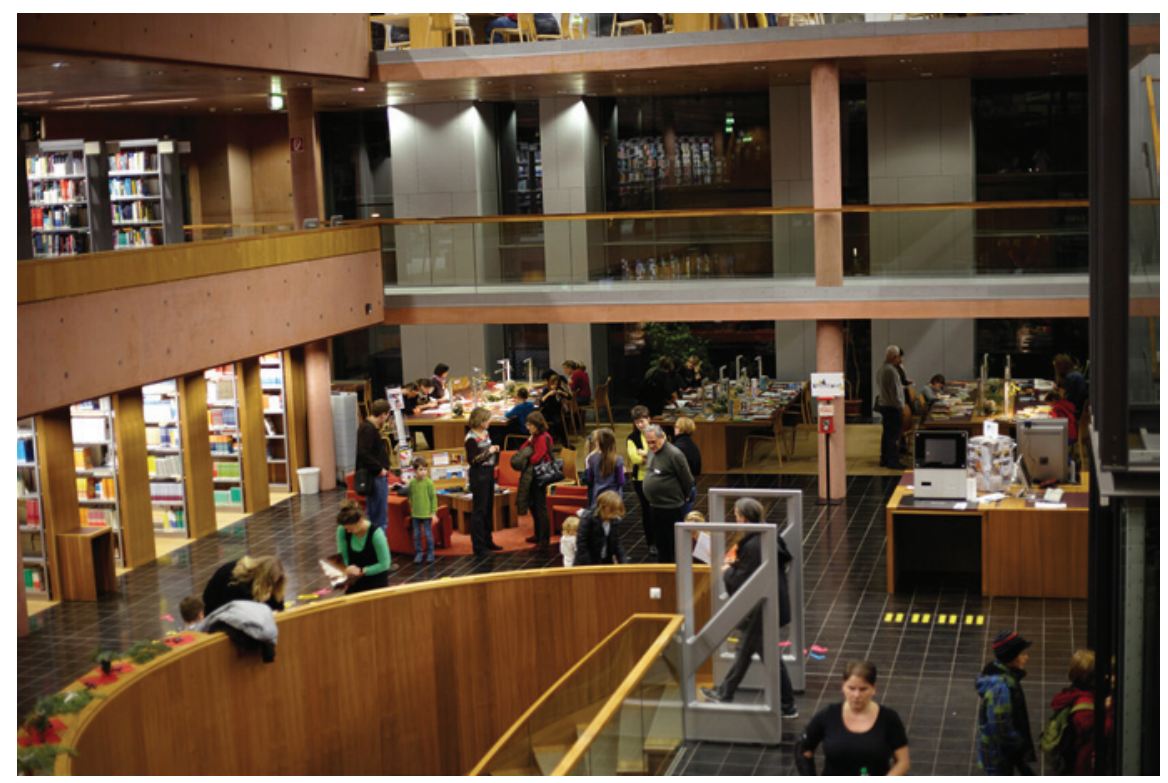

Abb. 2: Ein Blick auf die 1. Etage.

Im Bereich des Marketings wurde größtenteils an der alten Struktur festgehalten. Durch die gleiche Gestaltung der Werbematerialien ergab sich ein Wiedererkennungseffekt für die Veranstaltung. Die Öffentlichkeit wurde durch die regionale Pressearbeit und die Präsenz auf der Hochschulwebseite informiert. 
Konkrete Zielgruppen wurden definiert, um das Marketingkonzept weiter auszubauen. Bei der Planung sollten speziell Schulen angesprochen werden, um das Interesse der Lehrer und Schüler für den Schmökerabend und die Bibliothek anzuregen.

Aufgrund des hohen Aufwands und der guten Organisation wurde der Schmökerabend auf Seiten der Teilnehmer und der Hochschulbibliothek ein großer Erfolg. Die Besucher fragten gleich nach dem Termin des nächsten Schmökerabends. Dieses Veranstaltungsformat konnte durch den großen Raum der Hochschulbibliothek sehr gut umgesetzt werden. Die Idee, dass die Räumlichkeiten einer wissenschaftlichen Bibliothek nicht nur den Hochschulangehörigen dienen, sondern einem breiteren Publikum offen stehen, wurde erfolgreich vermittelt.

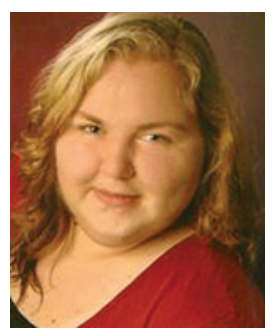

\author{
Nicole Kusserow \\ Auszubildende \\ Technische Hochschule Wildau (FH) \\ Hochschulring 1 \\ 15745 Wildau \\ Telefon: 03375-508548 \\ E-Mail: nicole.kusserow@th-wildau.de

\section{Pit Oertel} \\ Projektmitarbeiter \\ Technische Hochschule Wildau (FH) \\ Hochschulring 1 \\ 15745 Wildau \\ Telefon: 03375-508123 \\ E-Mail: pit.oertel@th-wildau.de
}

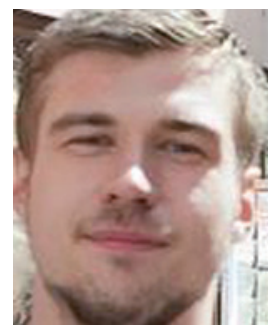

